ORIENTAL JOURNAL OF CHEMISTRY

An International Open Free Access, Peer Reviewed Research Journal

www.orientjchem.org
ISSN: 0970-020 X

CODEN: OJCHEG

2014, Vol. 30, No. (2):

Pg. 623-629

\title{
Thermodynamic Investigation of Tripeptide Tyr-Aaa-Gly in Gas Phase in Different Solvents
}

\author{
EBRAHIM BALALI*
}

Department of Chemistry, College of Basic Sciences, Tehran Science

and Research Branch, Islamic Azad University, Tehran, Iran.

*Corresponding author E-mail: e.balali93@gmail.com

http://dx.doi.org/10.13005/ojc/300230

(Received: April 04, 2014; Accepted: May 05, 2014)

\begin{abstract}
Tripeptide Tyr-Aaa-Gly $(1 \mathrm{a}, 2 \mathrm{~b})$ is methionine-enkephalin analogues $[\mathrm{Aaa}=(R, S)-(1-$ adamantyl)glycine]. The unique structure of adamantane is reflected in its highly unusual physical and chemical properties, which can have many applications including drug design and drug delivery. In this research, Quantum-mechanical calculations were performed at the HF/6-31G, $\mathrm{HF} / 6-31 \mathrm{G}^{*}$, B3LYP/6-31G, B3LYP/6-31+G, B3LYP/6-31G* and BLYP/6-31G** levels in the gas phase and four solvents such as water, DMSO, methanol and dichloromethane. According to these theoretical results we extracted thermo chemical parameters such as energy of the whole system, enthalpy, Gibbs free energy and entropy for Tripeptide Tyr-Aaa-Gly. The results were revealed that parameters are strongly affected by inducing different solvent media. According to these theoretical results it can be drastically concluded that the dielectric permittivity of the solvent is a key factor that determines the chemical behavior of Tripeptide Tyr-Aaa-Gly in solution.
\end{abstract}

Key words: Adamantane; Enkephalin; thermo chemical; Enthalpy; Gibbs free energy; entropy; gas phase; solvent effect.

\section{INTRODUCTION}

Adamantane is a highly lipophilic compounde, it is readily soluble in organic solvents, sublimes at $209-212{ }^{\circ} \mathrm{C}$, crystallizes at $-30^{\circ} \mathrm{C}$ and melts in sealed tubes at $268^{\circ} \mathrm{C}$. The unique structure of adamantane is reflected in its highly unusual physical and chemical properties, which can have many applications including drug design and drug delivery. The carbon skeleton of adamantine comprises a cage structure, which may be used for the encapsulation of other compounds, like drugs. Although adamantane has been the subject of many research projects in the field of pharmacophore-based drug design, its application to drug delivery and drug targeting systems is a new matter of considerable importance ${ }^{1}$.

Among the major biological activities displayed by adamantane derivatives, the 
antiviral $^{2}$, antibacterial ${ }^{3}$, antifungal ${ }^{4}$, antiinflammatory ${ }^{5}$, central nervous ${ }^{6}$ and $11 \mathrm{~b}-$ HSD1 inhibitory activities ${ }^{7}$ are the most important ones.

Due to the high lipophilicity of adamantane, the incorporation of the adamantyl moiety into several molecules results in compounds with relatively high lipophilicity, which in turn can modify the biological availability of these molecules.

Opioid peptides act as cell growth factors, in addition to regulating neurotransmission/ neuromodulation in the nervous system. To obtain more selective opioid peptides with improved or novel activity profiles toward malignant diseases, modifications using lipophilic moieties may be of particular benefit to passive or active cellular absorption by membrane penetration or attachment. The diastereomeric tripeptides Tyr-(S)-Aaa-Gly and Tyr- $(R)$-Aaa-Gly [Aaa = $(R, S)$-(1-adamantyl)glycine $]$ (Figure 1) showed similar, cell-selective, cytotoxic effects on tumor cell lines irrespective of Aaa chirality $^{8}$. They are methionine-enkephalin analogue.

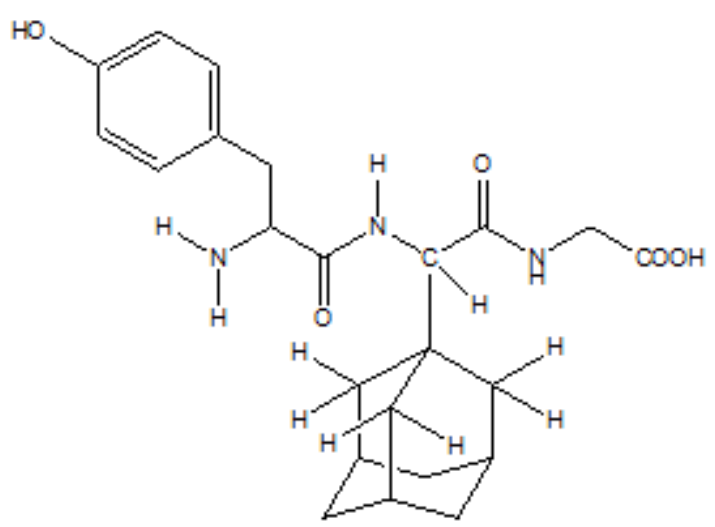

Fig. 1: Structure of Tripeptide Tyr-Aaa-Gly

Another example of adamantane utilization for poorly-absorbed-drug delivery to the brain is the conjugation of [D-Ala2]Leu-enkephalin derivatives with a 1-adamantyl. The antinociceptive effect of Leu-enkephalin disappears when it is administered peripherally since proteolytic enzymes would decompose it. As a result it cannot penetrate into the CNS (Central Nervous System). It is feasible to conjugate the [DAla2] Leu-enkephalin with a 1-adamantane vector via an ester, amide or a carbamate linkage in order to enhance the drug lipophilicity and thus facilitate its delivery across the blood-brain barrier (BBB) to the brain ${ }^{9}$. The adamantane-conjugated [D-Ala2] Leu-enkephalin prodrugs are highly lipophilic and show a significant antinociceptive effect because of their ability to cross the $\mathrm{BBB}^{10}$. These results suggest that adamantyl is a promising brain-directing drug vector providing a high lipophilicity, low toxicity and high BBB permeability for sensitive and poorly absorbed drugs $^{9,11}$.

\section{Methods of Computations}

At first, we have modeled the Tripeptide Tyr-Aaa-Gly (Figure 2) with ChemDraw package. Then using Chem3D performed an energy minimization. The ab initio molecular calculations were carried out using the Gaussian 98 program (Frisch et al., 1998) ${ }^{12}$. Gaussian is one of the most widely used quantum chemical program packages for molecular applications, and is used both in industry and in many scientific areas in academia. Geometry optimization in the gas phase and solution for molecules were performed at the HF method with the 6-31G, 6-31G* basis sets and at the B3LYP method with the $6-31 \mathrm{G}, 6-31+\mathrm{G}$ and $6-31 \mathrm{G}^{*}$ basis sets and BLYP method with the $6-31 \mathrm{G}^{* *}$ basis set in the gas phase and four solvents such as water, dimethylsulfoxide (DMSO), methanol and dichloromethane. The solvent effect is taken into account using the self-consistent reaction field (SCRF) method. This method is based on the Onsager reaction field theory of electrostatic salvation. In this model, a solvent is treated as a uniform dielectric with a given dielectric constant. A solute is placed into a cavity within the solvent. We optimized the geometries of Tripeptide Tyr-Aaa-Gly in various solvents using the Onsager model at the Hartree-Fock, BLYP and B3LYP levels of theory and compared our results with those obtained for the gas phase the effect of the permittivity of solvents on the stability of this structure. The energy of the whole system $(\Delta \mathrm{E})$, enthalpies $(\Delta \mathrm{H})$, Gibbs free energies $(\Delta G)$, entropies $(\Delta S)$ of Tripeptide Tyr-AaaGly were carried out in solution and gas phase. IR spectra studied in conjunction with normal mode calculations by quantum chemical methods provide details of conformational studies (Monajjemi et al., $2008)^{13}$. The first step of the calculations consisted of geometry optimization of the Tripeptide. SCRF approaches differ in how they define the cavity and reaction field (Monajjemi et al., 2010) ${ }^{14}$. 


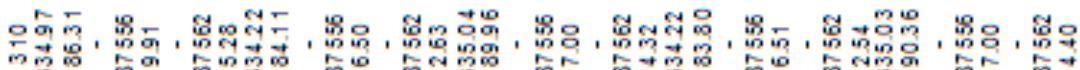

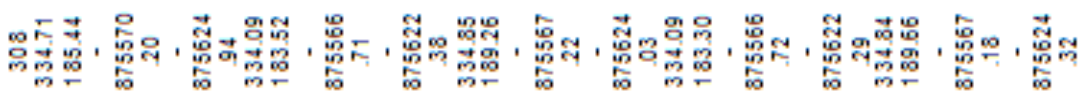

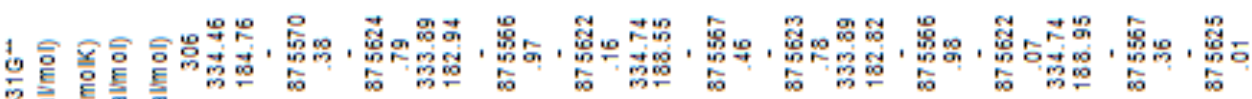

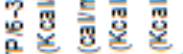

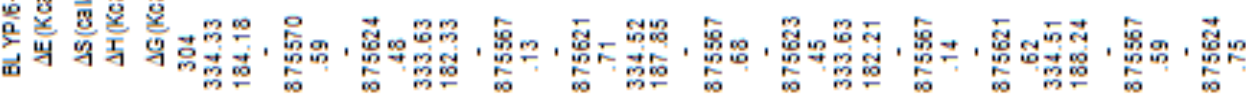

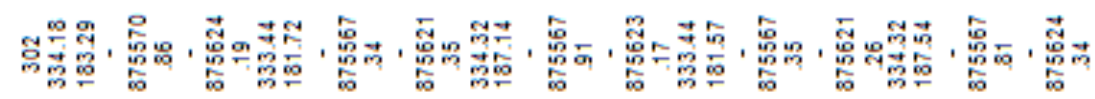

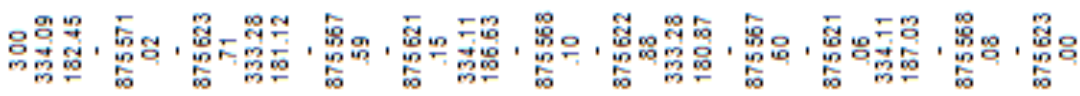

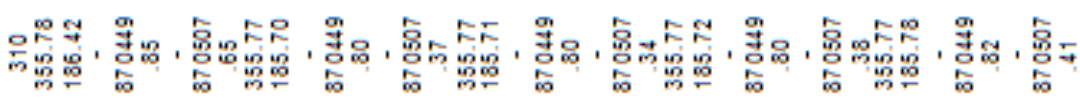

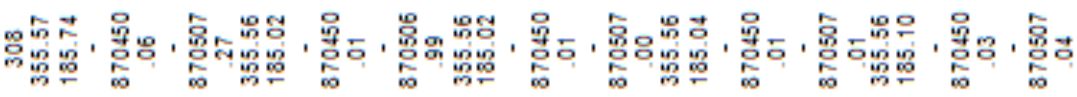

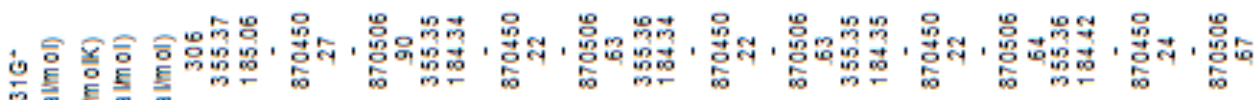
?

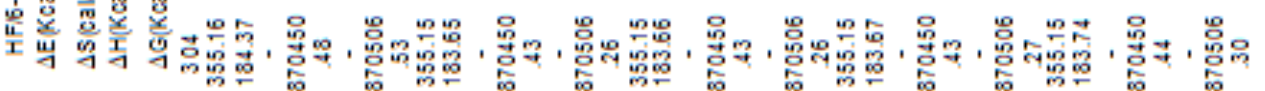

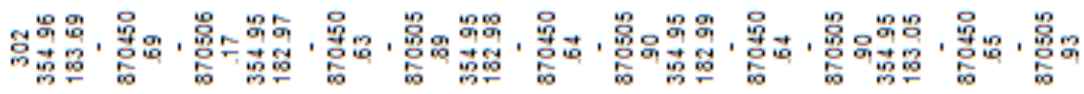

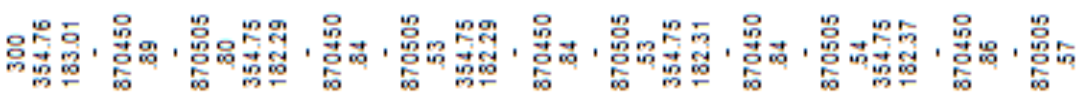

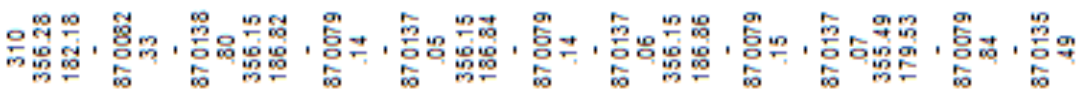

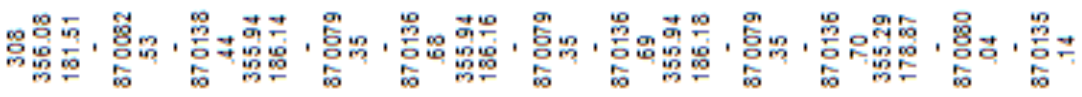

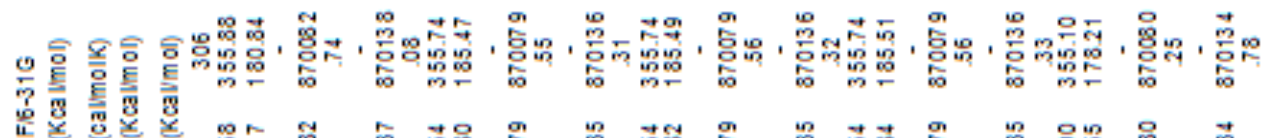

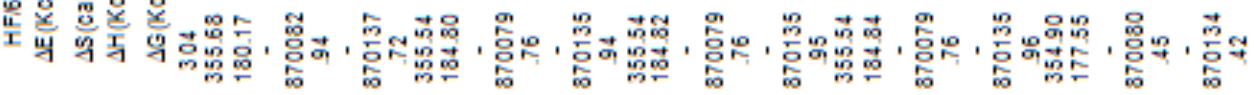

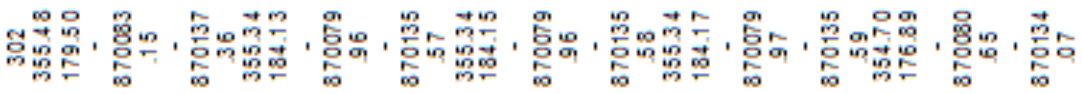

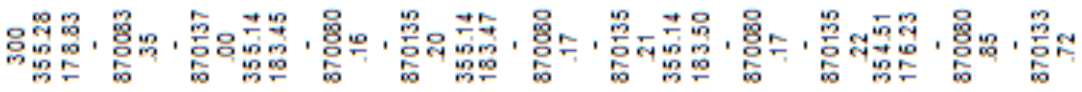

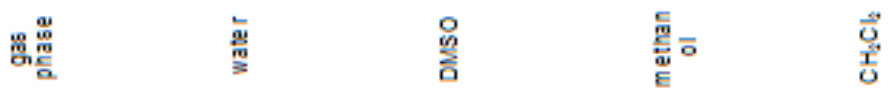




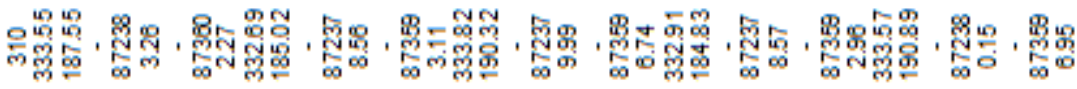

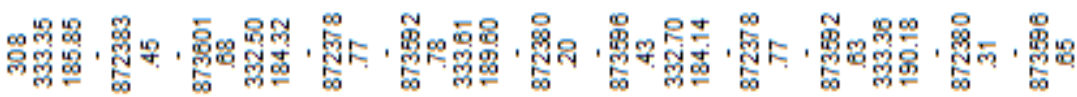

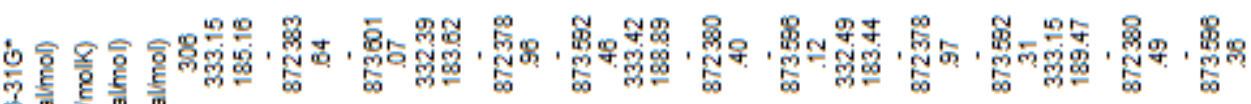

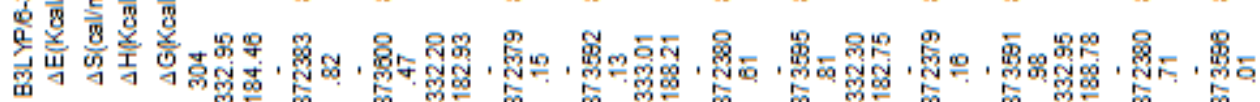

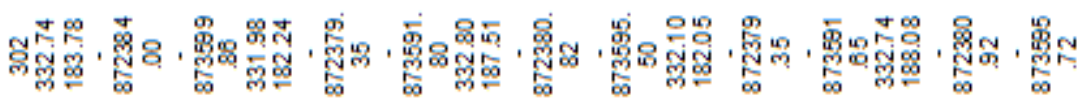

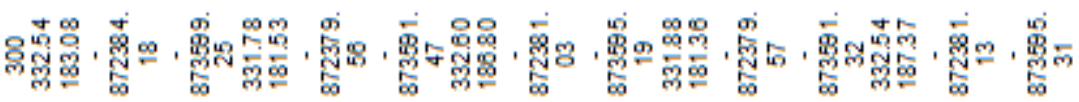

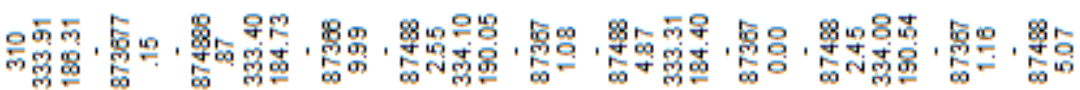

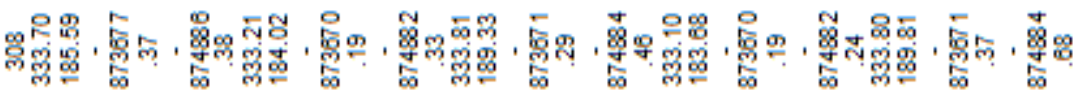

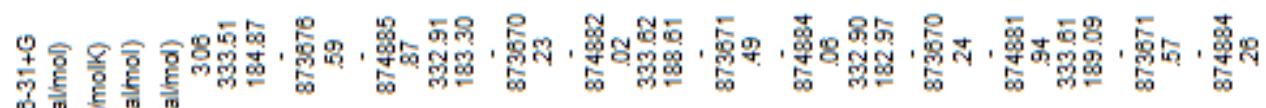
造要

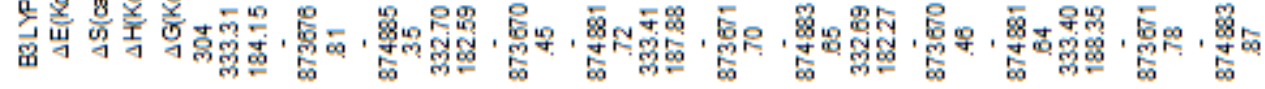

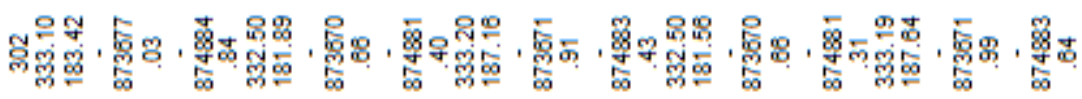

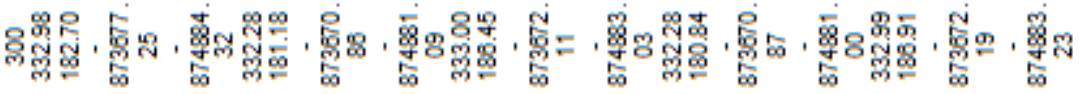

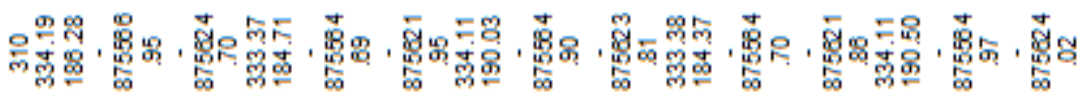

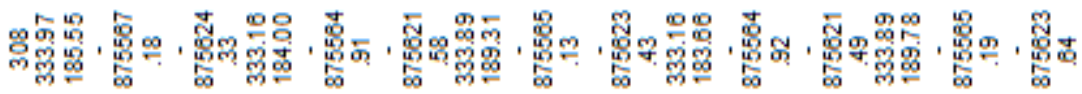

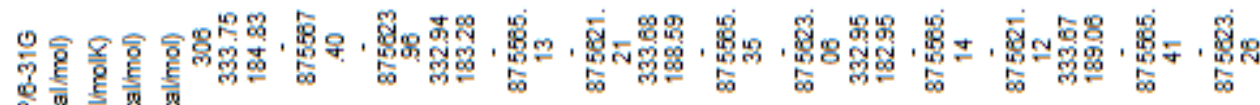

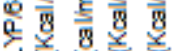

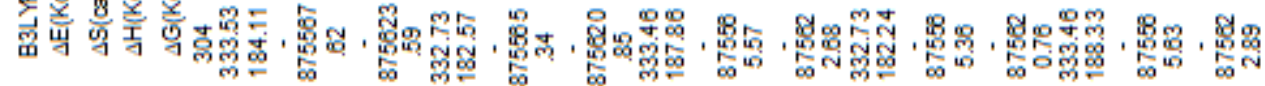

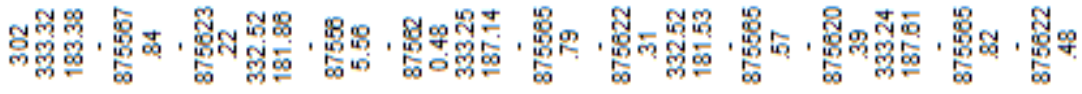

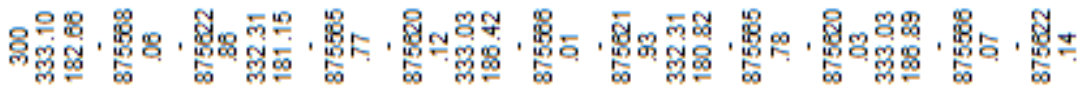

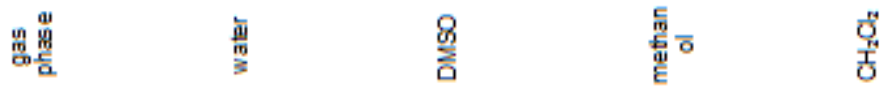




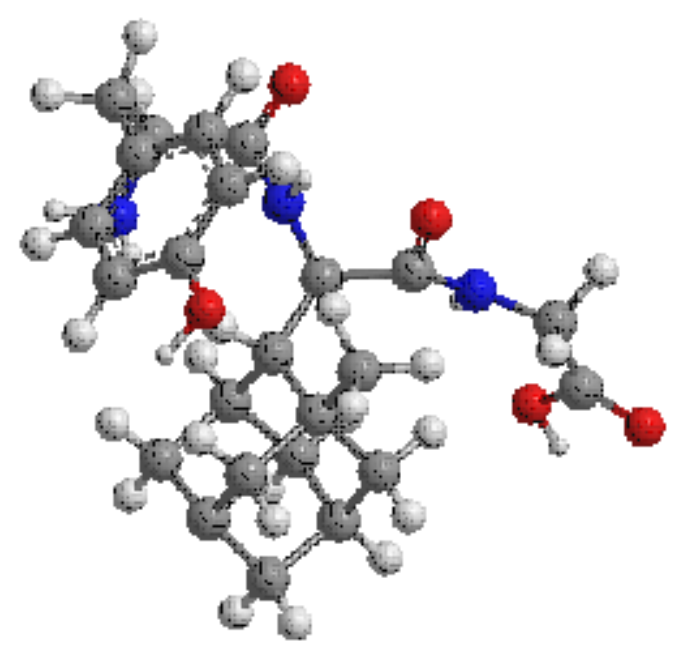

Fig. 2: Optimized structure of Tripeptide Tyr-Aaa-Gly.
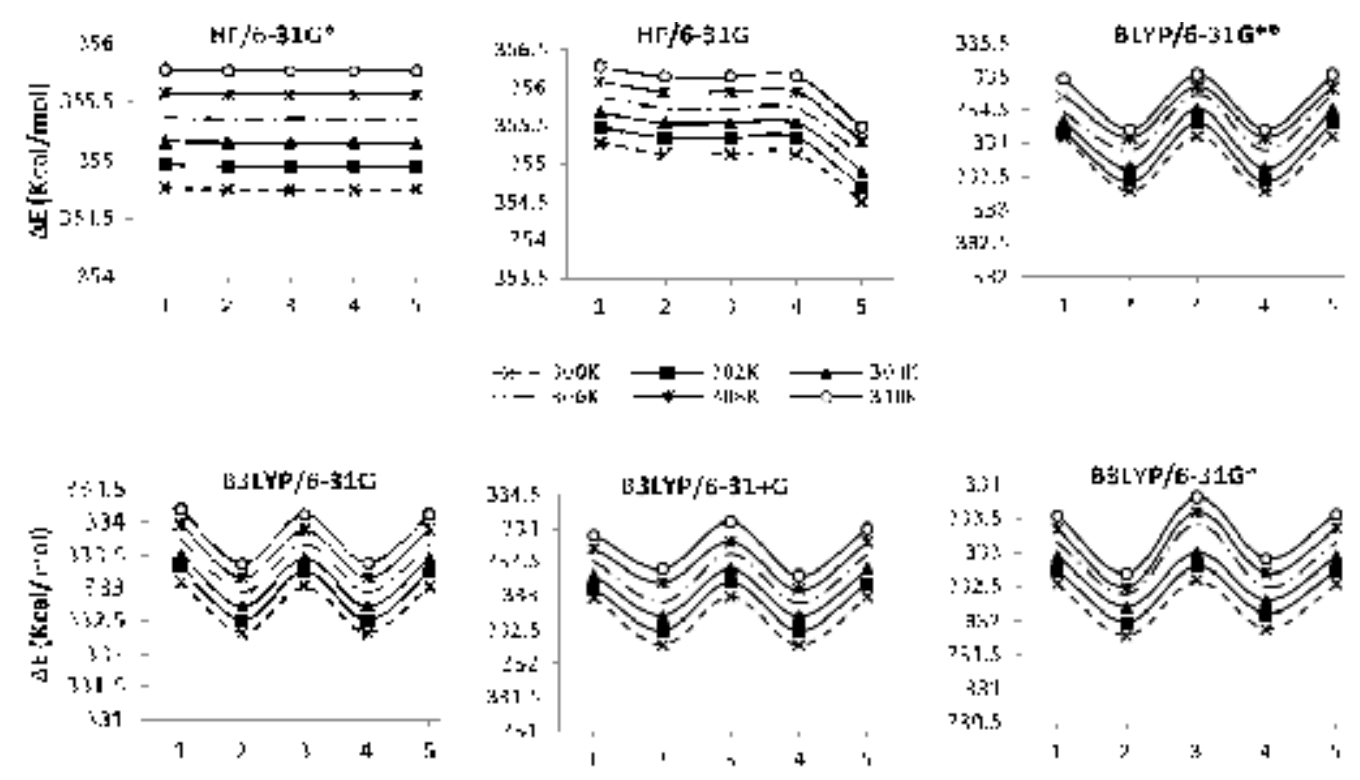

Fig. 3: Comparison of energy $(\Delta \mathrm{E})$ (Kcal/mol) of Tripeptide Tyr-Aaa-Gly in 1= gas phase, 2= water, 3= DMSO, 4= methanol and $5=\mathrm{CH}_{2} \mathrm{Cl}_{2}$ using the different levels at six temperatures $(300,302,304,306,308,310 \mathrm{~K})$

\section{DISCUSSION}

\section{Solvent effects on relative stabilities}

Tripeptide Tyr-Aaa-Gly (Figure 1) was studied in gas phase and various solvent media with dielectric constants of gas phase $(\mu=1)$, water $(\mu=80)$, dimethylsulfoxide $(\mu=46.7)$, methanol $(\mu$ $=32.63)$ and dichloromethane $(\mu=10.36)$. First, Tripeptide Tyr-Aaa-Gly was fully optimized by HF method with the 6-31G and 6-31G* basis sets and at the B3LYP method with 6-31G, 6-31+G and 6$31 \mathrm{G}^{*}$ basis set and BLYP method with $6-31 \mathrm{G}^{* *}$ basis set to obtain minima of the potential energy. The influence of the solvent on the relative stability of our molecule was studied by means of the Onsager approach (the SCRF method) at six temperatures 300, 302, 304, 306, 308 and 310 Kelvin (Table 1, 2). 
We found that there was some difference between these functions obtained by the different levels. As in Table 1, 2 and Figure 3 can be seen, in gas phase and solvents, the "E value increased with increasing of temperatures.

In fact at all medium (gas phase and different solvent) and using all levels, the "E value increased with increasing of temperatures. As in Figure 3 can be seen, using $\mathrm{HF} / 6-31 \mathrm{G}^{*}$ with change of medium don $i t$ change most. Using HF/6-31G level, " $E$ value in dichloromethane is the smallest value. The "E value, using four levels BLYP/6-31G**, $B 3 L Y P / 6-31 G$, B3LYP/6-31+G and B3LYP/6-31G* in water and methanol is smaller than other, while in gas phase, dimethylsulfoxide and dichloromethane is larger than other medium. The effect of solvents on the stabilization of the active site is of interest, it plays a major role in their activities. When solvent is added to Tripeptide TyrAaa-Gly the intermolecular hydrogen bonds are formed between Tripeptide and molecule of solvent. Water and methanol are protic solvents and from five sites with Tripeptide have interaction furthermore are formed strong hydrogen bonds. DMSO and dichloromethane have interaction with Tripeptide but intermolecular hydrogen bonds are not formed. We found that the relative energies ("E) of Tripeptide in solvents of water and methanol are smaller than in dimethylsulfoxide, dichloromethane and gas phase, because interactions in water and methanol are stronger than in dimethylsulfoxide and dichloromethan. On the other hand, interactions between water and methanol with Tripeptide reduce the energy of the whole system ("E). Also according to values listed in Table 1, 2, the largest value for " $E$ is watched using HF/6-31G level.

\section{Solvent effects on thermochemical parameters}

Thermochemical parameters such as standard enthalpy ("H), entropy ("S) and Gibbs free energy ("G) of Tripeptide Tyr-Aaa-Gly was obtained in gas phase and four solvent at six temperatures 300, 302, 304, 306, 308 and 310 Kelvin (Table 1, 2) using six levels. As in Table 1, 2 can be seen in gas phase and solvents, entropy ("S) value increased with increasing of temperature and the enthalpy (" $\mathrm{H})$ and Gibbs free energy ("G) of are negative values. We found that there was some difference between these functions obtained by the different levels. A study of hydrogen bonding between Tripeptide Tyr-Aaa-Gly and water, dimethylsulfoxide, methanol and dichloromethane was performed for optimized structure in solution (Table 1, 2).

According to Table 1, 2, we found that using $\mathrm{HF} / 6-31 \mathrm{G}^{*}$ level the largest value of "S observed in gas phase because in gas phase does not constitute. With HF/6-31G level in water, methanol and dimethylsulfoxide, the "S value is larger than in gas phase and dichloromethane. Also with four levels of BLYP/6-31G** , B3LYP/6-31G, B3LYP/6$31+G$ and $B 3 L Y P / 6-31 G^{*}$, the smallest value of "S belongs to water and methanol, while the greatest value belongs to dimethylsulfoxide, dichloromethane and gas phase. According to Table 1, 2, we found that using all methods, the most negative value of " $\mathrm{H}$ observed in gas phase. And finally as shown in Table 1, 2, the "G value using four levels BLYP/6-31G**, B3LYP/6-31G, $B 3 L Y P / 6-31+G$ and $B 3 L Y P / 6-31 G^{*}$ in gas phase, dimethylsulfoxide and dichloromethane is more negative than the other method. With two levels $\mathrm{HF} / 6-31 \mathrm{G}$ and HF/6-31G*, the most negative value is observed in gas phase.

\section{CONCLUSION}

In our current research, we have also theoretically studied the effects of water, DMSO, methanol, dichloromethane and gas phase at different temperatures on the thermodynamic parameters of Tripeptide Tyr-Aaa-Gly involving in active site and its structural stability. In order to investigate the influence different solvent media on the thermodynamic parameters through HF, BLYP and B3LYP methods and using 6-31G, 6-31G*, 6$31+G$ and $6-31 G^{\star *}$ basis sets. The best levels are $B L Y P / 6-31 G^{\star *}$, B3LYP/6-31G, B3LYP/6-31+G and $B 3 L Y P / 6-31 G^{*}$. In gas phase and solvents, entropy ("S) value and stability energy ("E) value increased with increasing of temperature. Also with increasing of temperature, the enthalpy (" $\mathrm{H})$ and Gibbs free energy ("G) of are negative values. On the basis of the results of our calculations, we found that the relative energies ("E) of Tripeptide Tyr-Aaa-Gly in solvents of water and methanol are smaller than in dimethylsulfoxide, dichloromethane and gas phase, because interactions in water and methanol are stronger than in dimethylsulfoxide and 
dichloromethan. On the other hand, interactions between water and methanol with Tripeptide reduce the energy of the whole system $(\Delta \mathrm{E})$. This Tripeptide, interactions in water and methanol are stronger than in dimethylsulfoxide and dichloromethane and gas phase, there for $\Delta S$ value in water and dichloromethane is larger than the other media. HF/
$6-31 \mathrm{G}^{*}$, the most negative value is observed in gas phase. According to our calculations, in the best methods, the most negative value of $\Delta \mathrm{H}$ observed in gas phase and the $\Delta G$ value in gas phase, dimethylsulfoxide and dichloromethane is more negative than the other method.

\section{REFERENCES}

1. Ramezani H., Mansoori G. A., Diamondoids as Molecular Building Block for Nanotechnology, Drug Targeting and Gene delivery, to appear 2006.

2. Wendel H. A., Snyder M. T., Pell S., Clin. Pharmacol. Therap., 1966; 7, 38-43.

3. Krimmel C. P., U.S. Patent, 1971; 3, 565.

4. Georgiev V. S., Bennett G. A., Radov L. A., Kamp D. K., Trusso L. A., Arch. Pharm., 1987; 320, 465-470.

5. Krimmel C. P., U.S. Patent 1968; 3, 374,244.

6. Schwab R. S., England A. C., Poskanzer D. C., Young R. R., J. Am. Med. Assoc., 1969; 208, 1168-1170.

7. Balkovec J. M., Thieringer R., Mundt S. S., Hermanowski-Vosatka A., Graham D. W., Donald W., Patel G. F., Susan S. D., Waddell S. T., Sherman T., Olson S. H., Maletic M., PCT Int. Appl. WO 20030814; Chem. Abstr. 2003; 139, 180065.

8. Horvat S., Mlinaric-Majerski K., Glavas-
Obrovac L., Jakas A., Veljkovic J., Marczi S., Kragol G., Roscic M., Matkovic M., MilosticSrb M., J. Med. Chem. 2006; 49, 3136-3142.

9. Tsuzuki N., Hama T., Hibi T., Konishi R., Futaki S., Kitagawa K. Biochemical Pharmacology. 41, R5-R8 1991.

10. Kazimierczuk Z., Gorska A., Switaj T., Lasek W., Bioorganic \& Medicinal Chem.Lett.s. 2001; 11: 1197-1200.

11. Kitagawa K.., Mizobuchi N., Hama T., Hibi T., Konishi R., Futaki S. Chem. \& Pharmaceutical Bulletin (Tokyo). 1997; 45, 1782-1787.

12. Frisch M. J. and et al GAUSSIAN 98, Revision A.6, Gaussian, Inc., Pittsburgh PA, 1998.

13. Monajjemi M., Rajaeian E., Mollaamin F., Naderi F., Saki S., Inter. J. Phys. Sciences, 2008; 5: 1450-1464.

14. Monajjemi M., Mahdavian L., Mollaamin F., Honarparvar B., Fullerenes, Nanotubes Carbon Nanostructures, 2010; 18, 45-55. 